Crossroads The ACM Magazine for Students
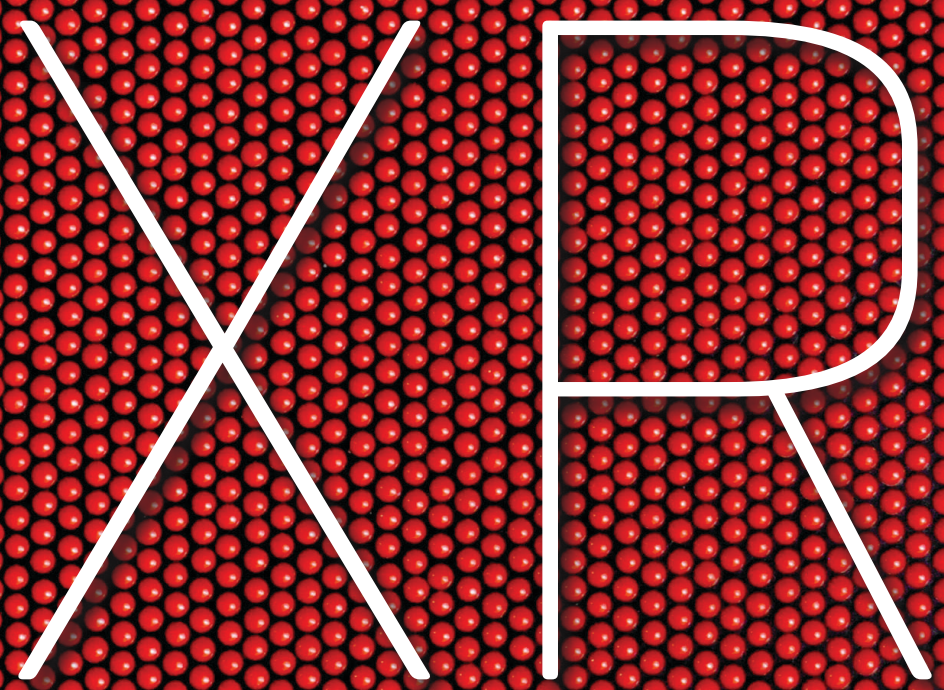

WINTER 2017

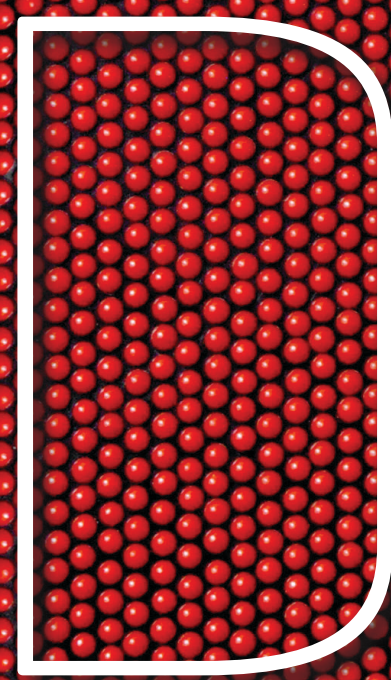

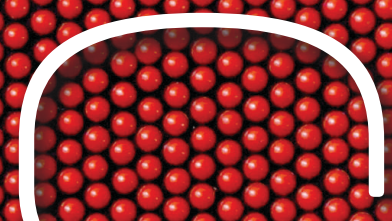

\title{
the
}

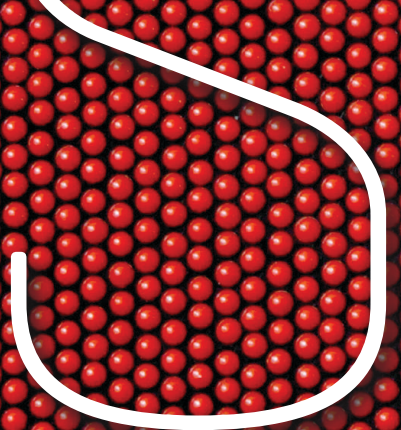

$-202020.50$

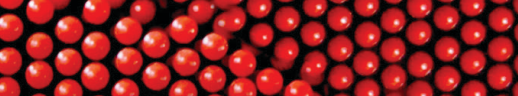

\section{Himan to}

Digitaland Physical Barriers toGhanging Identities

Identifying Hate Speech in Sócial Media

Gender and the Art of Community Relations 


\section{Robin Hammerman and Andrew L. Russell}

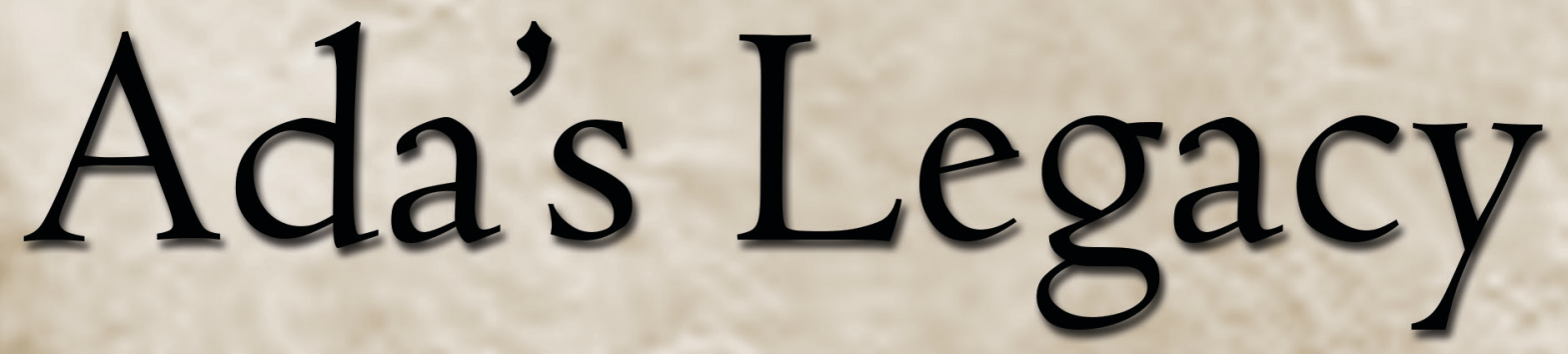

\section{Cultures of Computing from the Victorian to the Digital Age}

\section{Ada's Legacy}

Cultures of Computing from

the Victorian to the Digital Age

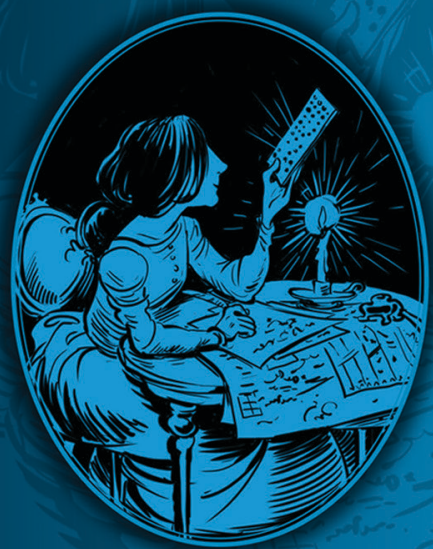

Robin Hammerman Andrew L. Russell

(iim) $\mathrm{MC}$

\section{INSPIRING MINDS FOR 200 YEARS}

Ada's Legacy illustrates the depth and diversity of writers, things, and makers who have been inspired by Ada Lovelace, the English mathematician and writer.

The volume commemorates the bicentennial of Ada's birth in December 1815, celebrating her many achievements as well as the impact of her work which reverberated widely since the late 19 th century. This is a unique contribution to a resurgence in Lovelace scholarship, thanks to the expanding influence of women in science, technology, engineering and mathematics.

ACM Books is a new series of high quality books for the computer science community, published by the Association for Computing Machinery with Morgan \& Claypool Publishers. 

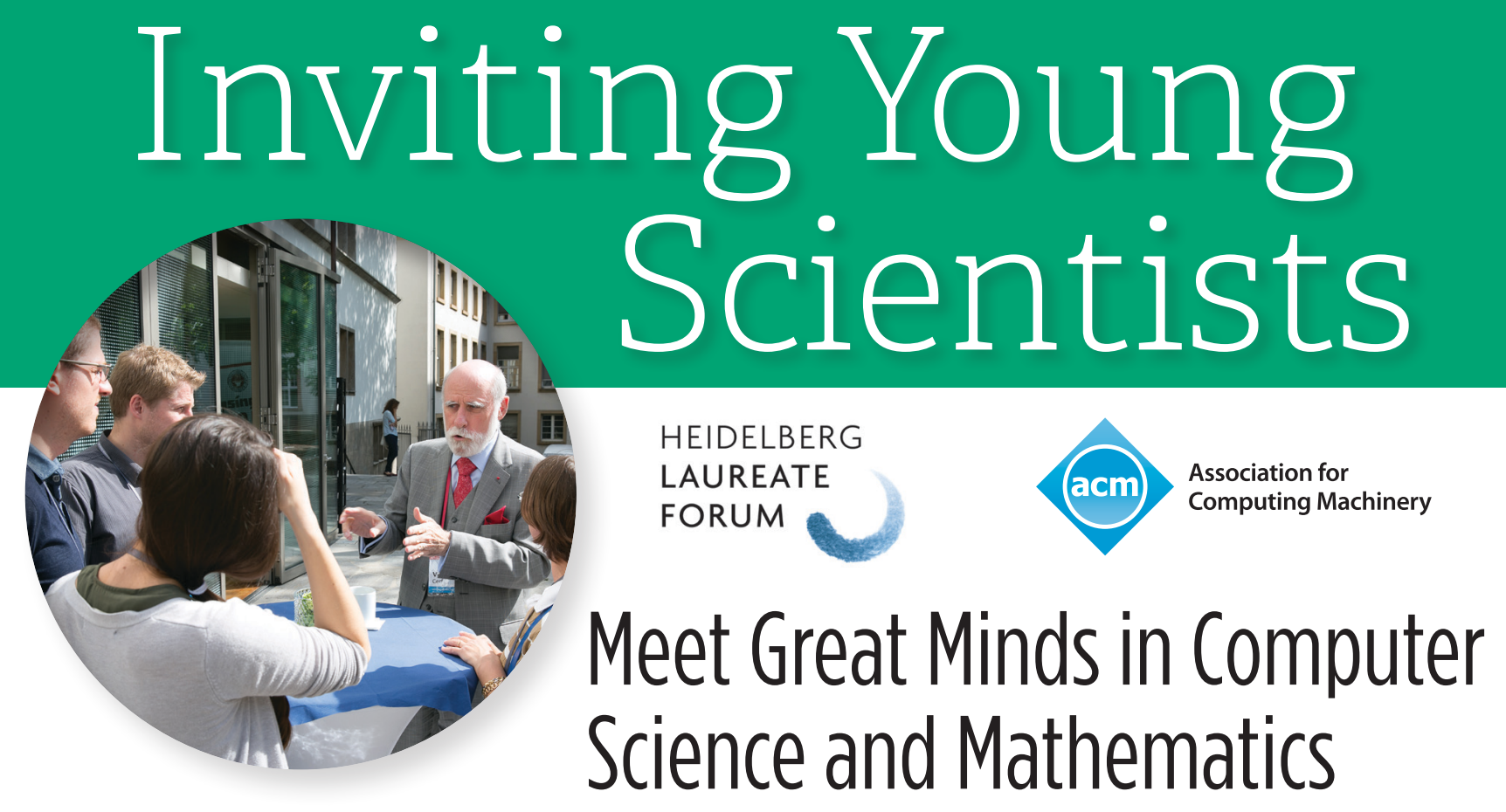

As one of the founding organizations of the Heidelberg Laureate Forum http://www.heidelberg-laureate-forum.org/, ACM invites young computer science and mathematics researchers to meet some of the preeminent scientists in their field. These may be the very pioneering researchers who sparked your passion for research in computer science and/or mathematics.

These laureates include recipients of the ACM A.M. Turing Award, the Abel Prize, the Fields Medal, and the Nevanlinna Prize.

The Heidelberg Laureate Forum is September 23-28, 2018 in Heidelberg, Germany.

This week-long event features presentations, workshops, panel discussions, and social events focusing on scientific inspiration and exchange among laureates and young scientists.

Who can participate?

New and recent Ph.Ds, doctoral candidates, other graduate students pursuing research, and undergraduate students with solid research experience and a commitment to computing research

How to apply:

Online: https://application.heidelberg-laureate-forum.org/ Materials to complete applications are listed on the site.

What is the schedule?

The application process is open between November 6, 2017 and February 9, 2018.

We reserve the right to close the application website early depending on the volume

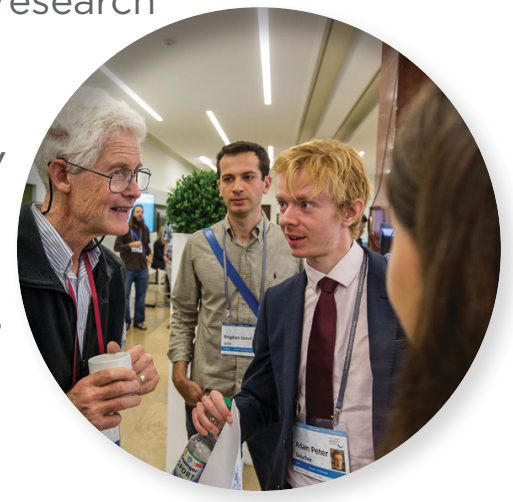
Successful applicants will be notified by mid April 2018.

More information available on Heidelberg social media 


\section{HUMAN TO HUMAN}

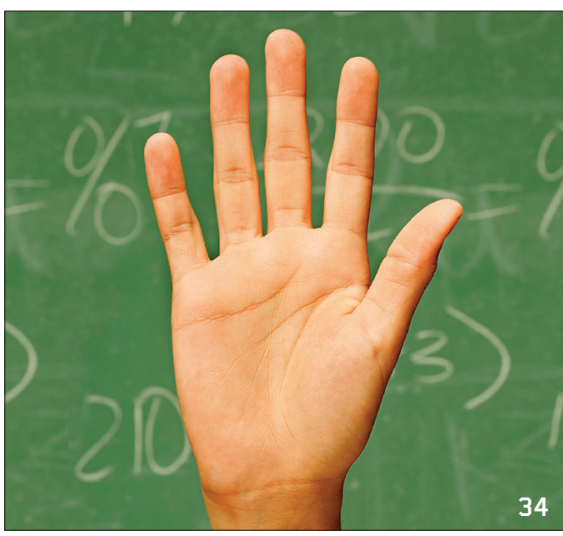

features

22 FEATURE

Can We Build the Cyborg Future

We All Deserve?

By Ari Schlesinger

\section{FEATURE}

Digital and Physical Barriers

to Changing Identities

By Oliver L. Haimson

\section{FEATURE}

Disability-Disclosure

Preferences and Practices in

Online Dating Communities

By Cynthia L. Bennett

\section{FEATURE}

Leveraging Personal Experience for Academic Research and Outreach ByJoslenne Peña

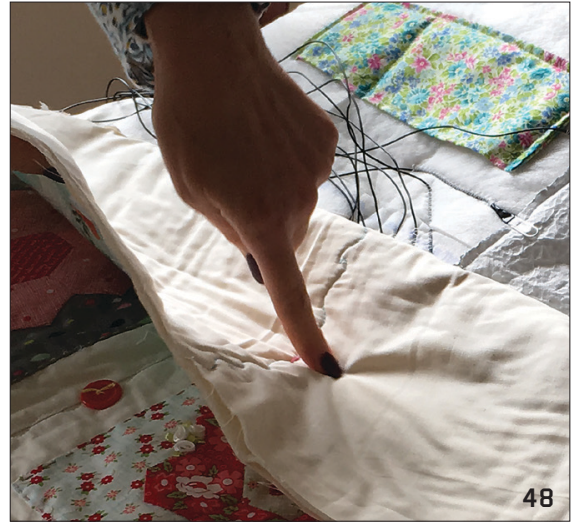

. end

\section{8}

FEATURE

Gender and the Art of Community Relations

ByLesley Mitchell

\section{FEATURE \\ Reflecting on Robots, \\ Love, and Poetry \\ By Margaret Rhee}

\begin{tabular}{l}
\hline 8 FEATURE \\
“We Had Tough Times, \\
But We've Sort Of Sewn Our Way \\
Through It:" The Partnership Quilt \\
By Angelika Strohmayer \\
and Janis Meissner
\end{tabular}

52 PROFILE

Sara Mauskopf

Innovative Thinking in

the Service of Parents

ByAdrian Scoică

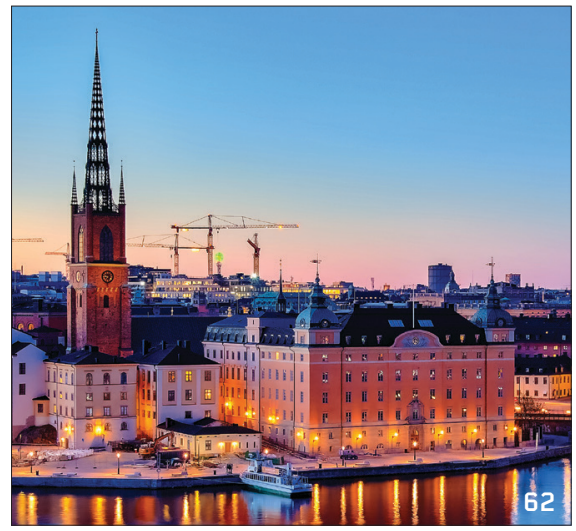

end

54 LABZ

Social and Psychological Questions about Humans and Technology

By David M. Markowitz

\section{BACK \\ Equality through \\ Digital Technology \\ By Vasileios Kalantzis}

\section{HELLO WORLD \\ Identifying Hate Speech \\ in Social Media \\ ByAlexandra Schofield \\ and Thomas Davidson}

\section{POINTERS}

\section{JARGON}

62 EVENTS

64 BEMUSEMENT 


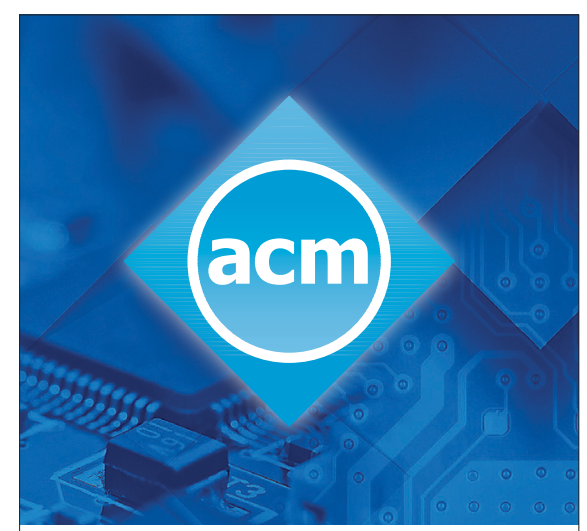

Advertise with ACM!

Reach the innovators and thought leaders working at the cutting edge of computing and information technology through ACM's magazines, websites and newsletters.

$$
\diamond \diamond \diamond \diamond \diamond
$$

\section{Request a media kit with specifications and pricing:}

\author{
Ilia Rodriguez
}

+1 212-626-0686 acmmediasales@acm.org

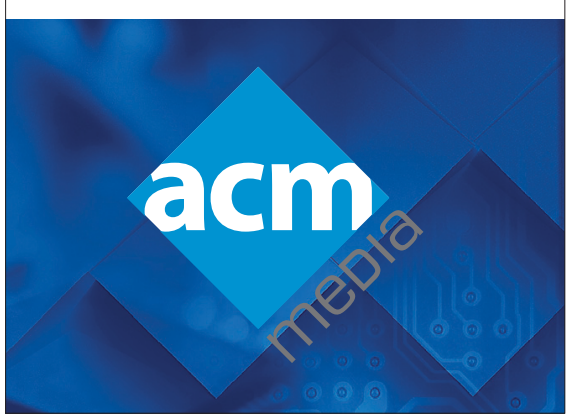

\section{XRDS}

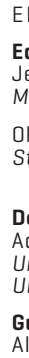
Editors-in-Chief
Jennifer Jacobs

Okke Schrijvers

Stanford University, USA

\section{Departments Chief}

Adrian Scoică

University of Cambridge, UK

Guest Editor

Alex Ahmed

Northeastern University, USA

Teresa Almeida

Newcastle University, UK

Issue Feature Editor

Judeth Oden Cho

Carnegie Mellon

University, USA

\section{Feature Editors}

Eliezer Bernart
Federal University of Rio

Federal University of Rio

Henri Maxime Demoulin University of Pennsylvania USA

Ophir Friedler

Tel Aviv University, Israel

Gunnar E. Wolf Iszaevich National Autonomous University of Mexico,

Mexico

Numair Khan

Brown University, USA

Talia Kohen

Bar Ilan University, Israel

Nidhi Rastogi

Rensselaer Polytechnic Institute, USA

Yang Shen

University of California,

Los Angeles, USA

\section{Department Editors}

Amity University, India

Abhishek Bhattacharya Amity University, India

Andrew J. Hunsucker Indiana University, USA

Vasileios Kalantzis University of Minnesota USA

Bryan Knowles Western Kentucky University, USA

Anshuman Majumdar International Institute of Information Technology, India

Darshit Patel

PimpriChinchwad College of Engineering, India

Alok Pandey

University of Petroleum and Energy Studies, India

Johanna Schacht Karlsruhe Institute of Technology, Germany
Digital Content Editor Pedro Lopes Hasso Plattner Institut. Germany

News Editors
Ankush Patak

Pimpri Chinchwad College of Engineering, India

\section{Bloggers}

David Guerra

Universitat de Lleida, Spain

Abdelrahman Hosny University of Connecticut USA

Andrew J. Hunsucker Indiana University, USA

Vasileios Kalantzis University of Minnesota, USA

Vassilios Karakoidas Athens University of

Economics and Business,

Greece

Maria Kechagia

Athens University of

Economics and Business,

Greece

Norene Kelly

lowa State University, USA

Pedro Lopes

Hasso Plattner Institut Germany

Dimitris Mitropoulos Athens University of

Economics and Business, Greece

Wolfgang Richter Carnegie Mellon

University, USA

Abhineet Saxena Guru Gobind Singh Indraprastha University, India

Gustavo Fortes Tondello University of Waterloo,

Canada
ADVISORY BOARD

Mark Allman,

International Computer Science Institute, USA

Bernard Chazelle,

Princeton University, USA

Laurie Faith Cranor,

Carnegie Mellon

University, USA

Alan Dix,

Lancaster University, UK

David Harel,

Weizmann Institute

of Science, Israel

Panagiotis Takis Metaxas, Wellesley College, USA

Noam Nisan, Hebrew

University Jerusalem,

Israel

Bill Stevenson, Apple, Inc., USA.

Andrew Tuson,

City University London, UK

Jeffrey D. Ullman,

Stanford University, USA

Moshe Y. Vardi,

Rice University, USA

EDITORIAL STAFF

Director of Publications Scott E. Delman

XRDS Managing Editor and Senior Editor at ACM HQ Denise Doig

Art Direction

Andrij Borys Associates, Andrij Borys.

Margaret Gray,

Mia Balaquiot

Advertising Sales

Account Manager

Ilia Rodriguez

ilia.rodriguez@acm.org

Copyright Permissions Deborah Cotton

permissions@acm.org

Editorial Assistant

Jade Morris

ACM

Association for

Computing Machinery

2 Penn Plaza,

Suite 701

New York, NY

10121-0701 USA

+1 212-869-7440

CONTACT

General feedback:

xrds@acm.org

For submission

quidelines, please see

http://xrds acm org/

authorguidelines.cfm

PUBLICATIONS BOARD

Co-Chairs

Jack Davidson and

Joseph A. Konstan

\section{Board Members}

Phoebe Ayers;

Anne Condon; Nikil Dutt:

Roch Guerrin; Chris Hankin;

Carol Hutchins:

Yannis loannidis; Sue Moon

Michael L. Nelson;

Sharon Oviatt:

Sharon Uviatt;

Eugene H. Spafford;
Stephen N. Spencer;

Alex Wade: Keith Webster:

Julie R. Williamson

SUBSCRIBE

Subscriptions [ $\$ 19$

per year includes $X R D S$ electronic subscription] are available

by becoming an

ACM Student Member

ACM Student Mem

www.acm.org/

Non-member

subscriptions:

$\$ 80$ per year

http://store.acm.org/

acmstore

\section{ACM Member Services}

To renew your ACM

membership or XRDS

subscription, please send

a letter with your name,

address, member number

and payment to:

ACM General Post Office

P.O. Box 3077

New York, NY

10087-0777 USA

Postal Information

XRDS [ISSN\# 1528-4981]

is published quarterly in

spring, winter, summer

and fall by Association fo

Computing Machinery,

2 Penn Plaza, Suite 701

New York, NY 10121.

Application to mail at

Periodical Postage rates

is paid at New York NY

and additional mailing

offices.

POSTMASTER: Send addresses change to:

XRDS: Crossroads,

Association for

Computing Machinery,

2 Penn Plaza, Suite 701

New York, NY 10121.

Offering\# XRDS0171

ISSN\# 1528-4972 [print]

ISSN\# 1528-4980

[electronic]

Copyright @2017 by

the Association for

Computing Machinery, Inc.

Permission to make digital

or hard copies of part of

this work for personal or

classroom use is granted

without fee provided that

copies are not made or 